\title{
ON THE TOTAL CURVATURE OF SURFACES IMMERSED IN EUCLIDEAN SPACES OF DIMENSION HIGHER THAN FOUR
}

\author{
YONG-SOO PYO AND YONG-TAE SHIN
}

\section{Introäuction}

The total absolute curvature $\tau(M)$ of a smoothly immersed $n$-submanifold $M$ of a Euclidean $(n+N)$-space $E^{n+N}$ was first studied by S.S.Chern and R.K.Lashof ([5]) and then by N.H.Kuiper ([6]) through the Lipschitz-Killing curvature $K(x, e)$ defined by the dual map $\tilde{\nu}^{*}$ of the Gauss map $\tilde{\nu}: B_{\nu} \rightarrow S_{0}^{n+N-1}$ of the unit normal bundle $B_{\nu}$ over $M$ into the unit $(n+N-1)$-sphere $S_{0}^{n+N-1}$ of the center at the origin in $E^{n+N}$ at each point $(x, e)$ of the bundle $B_{\nu}$ such that

$$
\tilde{\nu}^{*} d \sigma_{n+N-1}=K(x, e) d \sigma_{N-1} \Lambda d v
$$

where $d v$ and $d \sigma_{m}$ are the volume elements of $M$ and an $m$-sphere $S^{m}$, respectively.

The geometric meaning of the Lipschitz-Killing curvature $K(x, e)$ is described in detail by Y.T.Shin ([8]) as a generalization of the Gauss-Kronecker curvature of a hypersurface $M$ in $E^{n+1}$ or the Gauss curvature of $M$ in $E^{3}$. The total absolute curvature $K^{*}(x)=\int_{S^{N-1}}|K(x, e)| d \sigma_{N-1}$ at each point $x$ of $M$ is defined as the integral of the absolute value of the Lipschitz-Killing curvature $K(x, e)$ over each fiber of the unit normal bundle $B_{\nu}$ over $M$, and the total absolute curvature $\tau(M)=\int_{M} K^{*}(x) d v$ of $M$ as the integral of $K^{*}(x)$ over $M$ if it exists.

One of results Chern-Lashof and Kuiper proved in their first papers, applying the Morse inequality ([7]), is

$$
\tau(M) \geq C_{n+N-1} \beta(M),
$$

where $C_{n+N-1}$ is the volume of $(n+N-1)$-sphere $S^{n+N-1}$ and $\beta(M)$ is the sum of the betti numbers of $M$. The right-hand side of (1.2) depends on the coefficient field. If the equality sign holds in (1.2) with the real field as coefficient field, then $M$ has zero torsion. And we know the Gauss-Bonnet theorem for a compact surface $M$ in $E^{m}$

$$
\int_{M} G d v=2 \pi x(M)
$$

where $G$ is the Gauss curvature and $x(M)$ is the Euler characteristic of $M$.

This research is partially supported by the KOSEF, 1987-88. 
Some of the results we have proved in this paper are the following.

IN $M$ is a compact flat surface in $E^{m}$ with $\lambda_{2} \leq 0$, then we have

$$
\int_{M} \alpha^{2} d v \geq \frac{\pi^{2}}{2} \beta(M) .
$$

Let $M$ be a compact surface in $E^{5}$. Then we have

$$
\tau(M) \leq \frac{8}{3} \pi^{2} \chi(M)-8 \pi \int_{M} \lambda_{3}(x) d v .
$$

Concering the total absolute curvature $\tau(M)$ and the total mean curvature $\int_{M} \alpha^{2} d v$ of a compact surface in $E^{5}$ with $\lambda_{1} \geq 0$, we have proved the inequality

$$
\int_{M} \alpha^{2} d v \geq \frac{1}{24 \pi}\left(3 \tau(M)+8 \pi^{2} \chi(M)\right) .
$$

Let $M$ be a flat surface in $E^{5}$ with $\lambda_{2} \geq 0$. Then we have

$$
\int_{M} \alpha^{2} d v \geq \frac{3}{16 \pi} \tau(M) .
$$

\section{Preliminaries}

Let $M$ be a surface in a Euclidean $m$-space $E^{m}, m \geq 5$. We choose a local field of orthonormal frames $e_{1}, e_{2}, \ldots, e_{m}$ in $E^{m}$ such that, restricted to $M, e_{1}, e_{2}$ are tangent to $M$ and $e_{3}, \ldots, e_{m}$ are normal to $M$. Let $\omega^{1}, \omega^{2}, \cdots, \omega^{m}$ be the field of dual frame. Then the structure equations of $E^{m}$ are given by

$$
\begin{aligned}
d \omega^{A} & =-\sum_{B} \omega_{B}^{A} \Lambda \omega^{B}, \\
\omega_{B}^{A} & +\omega^{B}{ }_{A}=0, \\
d \omega_{B}^{A} & =-\sum_{C} \omega^{A}{ }_{C} \Lambda \omega^{C}{ }_{B}, \quad A, B, C=1,2, \cdots, m .
\end{aligned}
$$

We restrict these forms to $M$. Then $\omega^{3}=\cdots=\omega^{m}=0$.

Since

$$
0=d \omega^{r}=-\sum_{i} \omega^{r}{ }_{i} \Lambda \omega^{i}, \quad i, j, k=1,2, \quad r, s, t=3,4, \cdots, m
$$

by Cartan's lemma, we may write

$$
\omega_{i}^{r}=\sum_{j} h_{i j}^{r} \omega^{j}, \quad h_{i j}^{r}=h_{j i}^{r} .
$$


We call $h=\sum_{r, i, j} h_{i j}^{r} \omega^{i} \omega^{j} e_{r}$ the second fundamental form of $\dot{M}$. The mean curvature vector $H$ is given by

$$
H=\frac{1}{2} \sum_{r}\left(h_{11}^{r}+h_{22}^{r}\right) e_{r} .
$$

They are generalized cases of the surfaces in $E^{3}$.

If $H=0$, then $M$ is called a minimal surface. In [10], it is proved that there does not exist a closed minimal submanifold in a Euclidean space.

The Gauss curvature $G$ and the mean curvature $\alpha$ are defined respectively by

$$
\begin{aligned}
& G=\sum_{r=3}^{m}\left(h_{11}^{r} h_{22}^{r}-h_{12}^{r} h_{12}^{r}\right) \\
& \alpha=\frac{1}{2}\left(\sum_{r=3}^{m}\left(h_{11}^{r}+h_{22}^{r}\right)^{2}\right)^{\frac{1}{2}} .
\end{aligned}
$$

For a normal vector $e=\sum_{r=3}^{m} a_{r} e_{r}$ at $x$ in $M$, the second fundamental tensor $A(x, e)$ at $(x, e)$ is given by

$$
A(x, e)=\sum_{r=3}^{m} a_{r} h_{i j}^{r} .
$$

The Lipschitz-Killing curvature $K(x, e)$ is defined by

$$
\begin{aligned}
K(x, e) & =\operatorname{det}(A(x, e)) \\
& =\left(\sum_{r=3}^{m} a_{r} h_{11}^{r}\right)\left(\sum_{s=3}^{m} a_{s} h_{22}^{s}\right)-\left(\sum_{t=3}^{m} a_{t} h_{12}^{t}\right)^{2} .
\end{aligned}
$$

For each $x$ in $M$, we denote by $T_{x}^{\perp}$ the normal space at $x$. We define a linear mapping $\gamma$ from $T_{x}^{\perp}$ into the space of all symmetric matrices of order 2 by

$$
\gamma\left(\sum_{r=3}^{m} a_{r} e_{r}\right)=\sum_{r=3}^{m} a_{r} A\left(x, e_{r}\right) .
$$

Then, since $\operatorname{dim} \mathrm{T}_{\mathrm{x}}^{\perp}=\operatorname{dim} \operatorname{ker} \gamma+\operatorname{dim} \operatorname{Im} \gamma$,

$$
\operatorname{dim} \operatorname{ker} \gamma \geq \mathrm{m}-5 \text {. }
$$

We choose $e_{3}, e_{4}, \ldots, e_{m}$ at $x$ in such a way that $e_{6}, \ldots, e_{m} \in$ ker $\gamma$. Then for any unit normal vector $e=\sum_{r} \cos \theta_{r} e_{r}$ at $x$, the Lipschitz-Killing curvature $K(x, e)$ at $(x, e)$ is given by

$$
\begin{aligned}
K(x, e) & =\operatorname{det}(\mathbb{A}(\mathrm{x}, \mathrm{e})) \\
& =\operatorname{det}\left(\sum_{r=3}^{m} h_{i j}^{r} \cos \theta_{r}\right) \\
& =\left(\sum_{r=3}^{5} h_{11}^{r} \cos \theta_{r}\right)\left(\sum_{s=3}^{5} h_{22}^{s} \cos \theta_{s}\right)-\left(\sum_{t=3}^{5} h_{12}^{t} \cos \theta_{t}\right)^{2} .
\end{aligned}
$$


The right hand side of (2.3) is a quadratic form on $\cos \theta_{r}$. Hence, by choosing a suitable unit orthogonal normal vectors $e_{3}, e_{4}, e_{5}$ at $x$, we may write

$$
\begin{aligned}
K(x, e)= & \lambda_{1}(x) \cos ^{2} \theta_{3}+\lambda_{2}(x) \cos ^{2} \theta_{4}+\lambda_{3}(x) \cos ^{2} \theta_{5}, \\
& \lambda_{1}(x) \geq \lambda_{2}(x) \geq \lambda_{3}(x) .
\end{aligned}
$$

From now on, we choose such a frame unless otherwise stated.

From (2.1), we obtain

$$
G=\lambda_{1}+\lambda_{2}+\lambda_{3} \text { everywhere on } M \text {. }
$$

Let $C_{m}$ be the volume of the unit $m$-sphere $S^{m}$. Then we know that

$$
C_{m}=\frac{2 \pi^{\frac{1}{2}(m+1)}}{\Gamma\left(\frac{1}{2}(m+1)\right)}
$$

where $\Gamma$ is the Gamma function.

By spherical integration ([9]), we obtain the following equations (2.6) and (2.7).

$$
\int_{S^{m}}\left|\cos ^{2} \theta_{r}-\cos ^{2} \theta_{s}\right| d \sigma=\frac{2 C_{m+2}}{\pi^{2}}, \quad r \neq s,
$$

where $d \sigma$ is the volume element of the unit $m$-sphere $S^{m}$.

$$
\int_{S^{m}} \cos ^{2} \theta_{r} d \sigma=\frac{C_{m+2}}{2 \pi} .
$$

\section{Main Results}

We begin with the following lemma which is crucial for our argument.

Lemma 3.1. Let $M$ be a surface in $E^{m}, m \geq 5$. Then $\lambda_{3} \leq 0$ everywhere on $M$.

Proof. Let $e$ be a unit normal vector at $x \in M$ which is perpendicular to the mean curvature vector $H$. Then

$$
H \cdot e=\frac{1}{2} \sum_{r}\left(h_{11}^{r}+h_{22}^{r}\right) \cos \theta_{r}=0,
$$

where $e=\sum_{r} \cos \theta_{r} e_{r}$. Hence

$$
\begin{aligned}
\left(\sum_{r=3}^{5} h_{11}^{r} \cos \theta_{r}+\sum_{s=3}^{5} h_{22}^{s} \cos \theta_{s}\right)^{2}= & \left(\sum_{r=3}^{5} h_{11}^{r} \cos \theta_{r}\right)+\left(\sum_{s=3}^{5} h_{22}^{s} \cos \theta_{s}\right)^{2} \\
& +2\left(\sum_{r=3}^{5} h_{11}^{r} \cos \theta_{r}\right)\left(\sum_{s=3}^{5} h_{22}^{s} \cos \theta_{s}\right)=0 .
\end{aligned}
$$


Therefore the Lipschitz-Killing curvature $K(x, e)$ at $(x, e)$ is given by

$$
K(x, e)=\left(\sum_{r=3}^{5} h_{11}^{r} \cos \theta_{r}\right)\left(\sum_{s=3}^{5} h_{22}^{s} \cos \theta_{s}\right)-\left(\sum_{t=3}^{5} h_{12}^{t} \cos \theta_{t}\right)^{2} \leq 0
$$

Thus, from view points of (2.3), we complete the proof.

Theorem 3.2. Let $M$ be a compact surface in $E^{m}$ with $\lambda_{3}=0$. Then $M$ is homeomorphic to a 2-sphere.

Proof. Let $S_{x}$ be the unit hypersphere of $T_{x}^{\perp}$ and let $d \sigma$ be the volume element of $S_{x}$. From (2.3) and (2.7), we have

$$
\begin{aligned}
K^{*}(x) & =\int_{S_{x}}\left|\lambda_{1}(x) \cos ^{2} \theta_{3}+\lambda_{2}(x) \cos ^{2} \theta_{4}\right| d \sigma \\
& =\left(\lambda_{1}(x)+\lambda_{2}(x)\right) \frac{C_{m-1}}{2 \pi}=\frac{C_{m-1}}{2 \pi} G(x),
\end{aligned}
$$

where $C_{m-1}$ denotes the volume of the unit $(m-1)$-sphere. Hence the total absolute curvature $\tau(M)$ of $M$ is given by

$$
\begin{aligned}
\tau(M) & =\frac{C_{m-1}}{2 \pi} \int_{M} G(x) d v \\
& =C_{m-1} \chi(M) \geq C_{m-1} \beta(M)
\end{aligned}
$$

by (1.2) and (1.3), where $\chi(M)$ denotes the Euler characteristic of $M$. Therefore $\chi(M) \geq$ $\beta(M)$. Since $\chi(M) \leq \beta(M), \chi(M)=\beta(M)$. Thus, by the arguments in the inequality (1.2), $M$ has zero torsion and $\chi(M)=2$. Hence $M$ is homeomorphic to a 2-sphere.

Lemma 3.3. If $M$ is a compact flat surface in $E^{m}$ with $\lambda_{2} \leq 0$, then we have

$$
\int_{M} \lambda_{1}(x) d v \geq \frac{\pi^{2}}{2} \beta(M)
$$

Proof. Since $\lambda_{1}=-\lambda_{2}-\lambda_{3}$,

$$
K(x, e)=\lambda_{2}(x)\left(\cos ^{2} \theta_{4}-\cos ^{2} \theta_{3}\right)+\lambda_{3}(x)\left(\cos ^{2} \theta_{5}-\cos ^{2} \theta_{3}\right)
$$

Hence

$$
\begin{aligned}
K^{*}(x) & \leq-\lambda_{2}(x) \int_{S_{x}}\left|\cos ^{2} \theta_{4}-\cos ^{2} \theta_{3}\right| d \sigma-\lambda_{3}(x) \int_{S_{x}}\left|\cos ^{2} \theta_{5}-\cos ^{2} \theta_{3}\right| d \sigma \\
& =-\left(\lambda_{2}(x)+\lambda_{3}(x)\right) \frac{2 C_{m-1}}{\pi^{2}}=2 \lambda_{1}(x) \frac{C_{m-1}}{\pi^{2}}
\end{aligned}
$$


by (2.6). Therefore, by (1.2),

$$
C_{m-1} \beta(M) \leq \tau(M) \leq \frac{2 C_{m-1}}{\pi^{2}} \int_{M} \lambda_{1}(x) d v
$$

Thus

$$
\int_{M} \lambda_{1}(x) d v \geq \frac{\pi^{2}}{2} \beta(M)
$$

Lemma 3.4. Let $M$ be a surface in $E^{m}$. Then we have $\alpha^{2} \geq \lambda_{1}$.

Proof.

$$
\begin{aligned}
4 \alpha^{2}= & \left(h_{11}^{3}\right)^{2}+\left(h_{22}^{3}\right)^{2}+\left(h_{11}^{4}\right)^{2}+\left(h_{22}^{4}\right)^{2}+\left(h_{11}^{5}\right)^{2}+\left(h_{22}^{5}\right)^{2} \\
& \quad+2\left(h_{12}^{3}\right)^{2}+2\left(h_{12}^{4}\right)^{2}+2\left(h_{12}^{5}\right)^{2}+2 G \\
\geq & 2 h_{11}^{3} h_{22}^{3}-2 h_{11}^{4} h_{22}^{4}-2 h_{11}^{5} h_{22}^{5}+2\left(h_{12}^{3}\right)^{2}+2\left(h_{12}^{4}\right)^{2}+2\left(h_{12}^{5}\right)^{2}+2 G \\
\geq & 2 \lambda_{1}-2 \lambda_{2}-2 \lambda_{3}+2 G=4 \lambda_{1} .
\end{aligned}
$$

Theorem 3.5. Let $M$ be a compact flat surface in $E^{m}$ with $\lambda_{2} \leq 0$. Then we have

$$
\int_{M} \alpha^{2} d v \geq \frac{\pi^{2}}{2} \beta(M)
$$

Proof. By Lemma 3.3 and Lemma 3.4,

$$
\int_{M} \alpha^{2} d v \geq \int_{M} \lambda_{1}(x) d v \geq \frac{\pi^{2}}{2} \beta(M) .
$$

Lemma 3.6. Let $M$ be a surface in $E^{5}$. Then we have

$$
K^{*}(x) \leq \frac{4 \pi}{3} G(x)-8 \pi \lambda_{3}(x)
$$

The equality sign holds when and only when $\lambda_{1}(x)=\lambda_{2}(x)=\lambda_{3}(x)$ or $\lambda_{3}(x)=0$.

Proof. Since $\Sigma_{r=3}^{5} \cos ^{2} \theta_{r}=1$, by $(2.3)$,

$$
\begin{aligned}
K(x, e) & =\lambda_{1}(x) \cos ^{2} \theta_{3}+\lambda_{2}(x) \cos ^{2} \theta_{4}+\lambda_{3}(x)\left(1-\cos ^{2} \theta_{3}-\cos ^{2} \theta_{4}\right) \\
& =\left(\lambda_{1}(x)-\lambda_{3}(x)\right) \cos ^{2} \theta_{3}+\left(\lambda_{2}(x)-\lambda_{3}(x)\right) \cos ^{2} \theta_{4}+\lambda_{3}(x) .
\end{aligned}
$$

By (2.5) and (2.7),

$$
\int_{S_{x}} \cos ^{2} \theta_{r} d \sigma=\frac{C_{4}}{2 \pi}=\frac{4 \pi}{3} .
$$


Therefore

$$
\begin{aligned}
K^{*}(x) & \leq\left(\lambda_{1}(x)-\lambda_{3}(x)\right) \frac{4 \pi}{3}+\left(\lambda_{2}(x)-\lambda_{3}(x)\right) \frac{4 \pi}{3}-\lambda_{3}(x) 4 \pi \\
& =\frac{4 \pi}{3} G(x)-8 \pi \lambda_{3}(x) .
\end{aligned}
$$

If $K^{*}(x)=\frac{4 \pi}{3} G(x)-8 \pi \lambda_{3}(x)$, then

$$
|K(x, e)|=\left(\lambda_{1}(x)-\lambda_{3}(x)\right) \cos ^{2} \theta_{3}+\left(\lambda_{2}(x)-\lambda_{3}(x)\right) \cos ^{2} \theta_{4}-\lambda_{3}(x)
$$

for all $\theta_{3}, \theta_{4}$. Hence

$$
\lambda_{1}(x)=\lambda_{2}(x)=\lambda_{3}(x) \text { or } \lambda_{3}(x)=0 .
$$

The converse of this is trivial.

From Lemma 3.6 and (1.3), we obtain the following.

Theorem 3.7. Let $M$ be a compact surface in $E^{5}$. Then we have

$$
\tau(M) \leq \frac{8}{3} \pi^{2} \chi(M)-8 \pi \int_{M} \lambda_{3}(x) d v
$$

where $\chi(M)$ is the Euler characteristic of $M$.

Lemma 3.8. Let $M$ be a surface in $E^{5}$ with $\lambda_{1} \geq 0$. Then we have

$$
K^{*}(x) \leq 8 \pi \lambda_{1}(x)-\frac{4 \pi}{3} G(x)
$$

The equality sign holds when and only when $\lambda_{1}(x)=0$.

Proof. Since $\Sigma_{r=3}^{5} \cos ^{2} \theta_{r}=1$,

$$
\begin{aligned}
K(x, e) & =\lambda_{1}(x)\left(1-\cos ^{2} \theta_{4}-\cos ^{2} \theta_{5}\right)+\lambda_{2}(x) \cos ^{2} \theta_{4}+\lambda_{3}(x) \cos ^{2} \theta_{5} \\
& =\lambda_{1}(x)+\left(\lambda_{2}(x)-\lambda_{1}(x)\right) \cos ^{2} \theta_{4}+\left(\lambda_{3}(x)-\lambda_{1}(x)\right) \cos ^{2} \theta_{5} .
\end{aligned}
$$

Hence

$$
\begin{aligned}
K^{*}(x) & \leq \lambda_{1}(x) C_{2}-\left(\lambda_{1}(x)+\lambda_{2}(x)+\lambda_{3}(x)\right) \frac{C_{4}}{2 \pi}+3 \lambda_{1}(x) \frac{C_{4}}{2 \pi} \\
& =8 \pi \lambda_{1}(x)-\frac{4 \pi}{3} G(x) .
\end{aligned}
$$

If the equality holds, then we must have

$$
|K(x, e)|=\lambda_{1}(x)+\left(\lambda_{1}(x)-\lambda_{2}(x)\right) \cos ^{2} \theta_{4}+\left(\lambda_{1}(x)-\lambda_{3}(x)\right) \cos ^{2} \theta_{5}
$$

for all $\theta_{4}$ and $\theta_{5}$. Therefore

$$
\lambda_{1}(x)=0 \text { or } \lambda_{1}(x)=\lambda_{2}(x)=\lambda_{3}(x) .
$$


But the second condition also implies $\lambda_{1}(x)=0$, since $\lambda_{1}(x) \geq 0$ and $\lambda_{3}(x) \leq 0$. The converse of this is trivial.

Theorem 3.9. Let $M$ be a compact surface in $E^{5}$ with $\lambda_{1} \geq 0$. Then we have

$$
\int_{M} \alpha^{2} d v \geq \frac{1}{24 \pi}\left(3 \tau(M)+8 \pi^{2} \chi(M)\right) .
$$

Proof. From Lemma 3.4 and Lemma 3.8,

$$
\begin{aligned}
\int_{M} \alpha^{2} d v & \geq \int_{M} \lambda_{1}(x) d v \\
& \geq \frac{1}{8 \pi} \int_{M}\left(K^{*}(x)+\frac{4 \pi}{3} G(x)\right) d v \\
& =\frac{1}{8 \pi}\left(\tau(M)+\frac{8 \pi^{2}}{3} \chi(M)\right) .
\end{aligned}
$$

Lemma 3.10. Let $M$ be a surface in $E^{m}$. Then we have

$$
\alpha^{2} \geq \lambda_{1}+\lambda_{2}
$$

Proof.

$$
\begin{aligned}
4 \alpha^{2}= & \left(h_{11}^{3}\right)^{2}+\left(h_{22}^{3}\right)^{2}+\left(h_{11}^{4}\right)^{2}+\left(h_{22}^{4}\right)^{2}+\left(h_{11}^{5}\right)^{2}+\left(h_{22}^{5}\right)^{2} \\
& \quad+2\left(h_{12}^{3}\right)^{2}+2\left(h_{12}^{4}\right)^{2}+2\left(h_{12}^{5}\right)^{2}+2 G \\
\geq & 2 h_{11}^{3} h_{22}^{3}+2 h_{11}^{4} h_{22}^{4}-2 h_{11}^{5} h_{22}^{5}+2\left(h_{12}^{3}\right)^{2}+2\left(h_{12}^{4}\right)^{2}+2\left(h_{12}^{5}\right)^{2}+2 G \\
\geq & 2 \lambda_{1}+2 \lambda_{2}-2 \lambda_{3}+2 G \\
= & 4\left(\lambda_{1}+\lambda_{2}\right) .
\end{aligned}
$$

Lemma 3.11. Let $M$ be a surface in $E^{5}$ with $\lambda_{2} \geq 0$. Then we have

$$
K^{*}(x) \leq 4 \pi \lambda_{2}(x)+\frac{4 \pi}{3}\left(\lambda_{1}(x)-\lambda_{3}(x)\right) .
$$

The equality sign holds when and only when $\lambda_{1}(x)=\lambda_{2}(x)=0$ or $\lambda_{2}(x)=\lambda_{3}(x)=0$.

Proof. $\quad K(x, e)=\lambda_{1}(x) \cos ^{2} \theta_{3}+\lambda_{2}(x)\left(1-\cos ^{2} \theta_{3}-\cos ^{2} \theta_{5}\right)+\lambda_{3}(x) \cos ^{2} \theta_{5}$

$$
=\lambda_{2}(x)+\left(\lambda_{1}(x)-\lambda_{2}(x)\right) \cos ^{2} \theta_{3}+\left(\lambda_{3}(x)-\lambda_{2}(x)\right) \cos ^{2} \theta_{5} .
$$

Hence

$$
\begin{aligned}
K^{*}(x) & \leq \lambda_{2}(x) C_{2}+\left(\lambda_{1}(x)-\lambda_{2}(x)\right) \frac{4 \pi}{3}+\left(\lambda_{2}(x)-\lambda_{3}(x)\right) \frac{4 \pi}{3} \\
& =4 \pi \lambda_{2}(x)+\frac{4 \pi}{3}\left(\lambda_{1}(x)-\lambda_{3}(x)\right) .
\end{aligned}
$$


If $K^{*}(x)=4 \pi \lambda_{2}(x)+\frac{4 \pi}{3}\left(\lambda_{1}(x)-\lambda_{3}(x)\right)$, then

$$
|K(x, e)|=\lambda_{2}(x)+\left(\lambda_{1}(x)-\lambda_{2}(x)\right) \cos ^{2} \theta_{3}+\left(\lambda_{2}(x)-\lambda_{3}(x)\right) \cos ^{2} \theta_{5}
$$

for all $\theta_{3}, \theta_{5}$. Therefore

$$
\lambda_{1}(x)=\lambda_{2}(x)=0 \text { or } \lambda_{2}(x)=\lambda_{3}(x) .
$$

But the second condition implies $\lambda_{2}(x)=\lambda_{3}(x)=0$, since $\lambda_{2}(x) \geq 0$ and $\lambda_{3}(x) \leq 0$. The converse of this trivial.

Theorem 3.12. Let $M$ be a flat surface in $E^{5}$ with $\lambda_{2} \geq 0$. Then we have $\int_{M} \alpha^{2} d v \geq \frac{3}{16 \pi} \tau(M)$.

Proof. Since $\lambda_{3}=-\lambda_{1}-\lambda_{2}$,

$$
\begin{aligned}
K^{*}(x) & \leq 4 \pi \lambda_{2}(x)+\frac{4 \pi}{3}\left(2 \lambda_{1}(x)+\lambda_{2}(x)\right) \\
& =\frac{8 \pi}{3}\left(\lambda_{1}(x)+2 \lambda_{2}(x)\right) \\
& =\frac{16 \pi}{3}\left(\lambda_{1}(x)+\lambda_{2}(x)\right)-\frac{8 \pi}{3} \lambda_{1}(x),
\end{aligned}
$$

by Lemma 3.11. From Lemma 3.10,

$$
\begin{aligned}
\int_{M} \alpha^{2} d v & \geq \int_{M}\left(\lambda_{1}(x)+\lambda_{2}(x)\right) d v \\
& \geq \frac{3}{16 \pi} \int_{M}\left(K^{*}(x)+\frac{8 \pi}{3} \lambda_{1}(x)\right) d v \\
& \geq \frac{3}{16 \pi} \tau(M), \text { since } \lambda_{1}(x) \geq 0
\end{aligned}
$$

\section{References}

[1] B.Y. Chen, On the total curvature of immersed manifolds I, II, III, IV, V, and VI, Amer. J. Math. 93(1971), 148-162; Amer. J. Math. 94(1972), 799-809; Amer.J.Math. 95(1973), 636-642; Bull. Inst. Math. Acad. Sinica 7(1979); Bull. Inst. Matth, Acad. Sinica 9(1981); 509-516; Bull. Inst. Math. Acad. Sinica 11(1983), 309-328.

[2] - An invariant of conformal mappings, Proc.A mer.Math.Soc. 40(1973), 563-564.

[3] - Total mean curvature of immersed surfaces in $E^{m}$, Trans. Amer. Math. Soc. 218(1976), 333-341.

[4] - Total mean curvature and submanifolds of finite type, World Scientific (1984), Singapore.

[5] S.S. Chern and R.K.Lashof, On the total curvature of immersed manifolds I and II, Amer.J.Math. 79(1957), 306-318; Michigan Math.J. 5(1958), 5-12.

[6] N.H. Kuiper, Immersions with minimal total absolute curvature, Coll. de Gèomètrie Differentielle Globale (Bruxelles, 1958), 75-88.

[7] J.Milnor, Morse Theory, Princeton Univ. Press\#51 (1963). 
[8] Y.T. Shin, On the total curvature of immersions, Thesis Coll.Chungnam Univ. Natural Sciences $13(2)(1974), 7-18$.

[9] —, and Y.S.Pyo, A derivation of some spherical integral formulas, J.of Keimyung Math Sci. $6(1986), 1-8$.

[10] T.J. Willmore, Total curvature in Riemannian geometry, Clarendon (1982).

Department of Applied mathematices, National Fisheries University of Pusan, Pusan 608-737, Koera. Department of Mathematices, Keimyung University, Taegu 704-701, Koera. 